\title{
Detailed volumetric analysis of the hypothalamus in behavioral variant frontotemporal dementia
}

\author{
Martina Bocchetta ${ }^{1,2,3} \cdot$ Elizabeth Gordon $^{1} \cdot$ Emily Manning $^{1} \cdot$ Josephine Barnes $^{1}$ • \\ David M. Cash ${ }^{1} \cdot$ Miklos Espak $^{1,4} \cdot$ David L. Thomas ${ }^{1}$ - Marc Modat ${ }^{1,4}$. \\ Martin N. Rossor ${ }^{1} \cdot$ Jason D. Warren $^{1} \cdot$ Sebastien Ourselin $^{1,4} \cdot$ Giovanni B. Frisoni $^{2,5}$. \\ Jonathan D. Rohrer ${ }^{1}$
}

Received: 1 June 2015/Revised: 18 August 2015/Accepted: 18 August 2015/Published online: 4 September 2015 (C) The Author(s) 2015. This article is published with open access at Springerlink.com

\begin{abstract}
Abnormal eating behaviors are frequently reported in behavioral variant frontotemporal dementia (bvFTD). The hypothalamus is the regulatory center for feeding and satiety but its involvement in bvFTD has not been fully clarified, partly due to its difficult identification on MR images. We measured hypothalamic volume in 18 patients with bvFTD (including $9 M A P T$ and 6 C9orf72 mutation carriers) and 18 cognitively normal controls using a novel optimized multimodal segmentation protocol, combining 3D T1 and T2-weighted 3T MRIs (intrarater intraclass correlation coefficients $\geq 0.93$ ). The whole hypothalamus was subsequently segmented into five subunits: the anterior (superior and inferior), tuberal (superior and inferior), and posterior regions. The presence of abnormal eating behavior was assessed with the revised version of the Cambridge Behavioural Inventory (CBI-R).
\end{abstract}

Electronic supplementary material The online version of this article (doi:10.1007/s00415-015-7885-2) contains supplementary material, which is available to authorized users.

Jonathan D. Rohrer

j.rohrer@ucl.ac.uk

1 Dementia Research Centre, Department of Neurodegenerative Disease, UCL Institute of Neurology, 8-11 Queen Square, London WC1N 3BG, UK

2 Laboratory of Alzheimer's Neuroimaging and Epidemiology, IRCCS Istituto Centro San Giovanni di Dio Fatebenefratelli, Brescia, Italy

3 Department of Molecular and Translational Medicine, University of Brescia, Brescia, Italy

4 Translational Imaging Group, Centre for Medical Image Computing, University College London, London, UK

5 Memory Clinic and Laboratory of Neuroimaging of Aging, University Hospitals and University of Geneva, Geneva, Switzerland
The bvFTD group showed a $17 \%$ lower hypothalamic volume compared with controls $(p<0.001)$ : mean 783 (standard deviation 113) versus $944(73) \mathrm{mm}^{3}$ (corrected for total intracranial volume). In the hypothalamic subunit analysis, the superior parts of the anterior and tuberal regions and the posterior region were significantly smaller in the bvFTD group compared with controls. There was a trend for a smaller hypothalamic volume, particularly in the superior tuberal region, in those with severe eating disturbance scores on the CBI-R. Differences were seen between the two genetic subgroups with significantly smaller volumes in the MAPT but not the C9orf72 group compared with controls. In summary, bvFTD patients had lower hypothalamic volumes compared with controls. Different genetic mutations may have a differential impact on the hypothalamus.

Keywords Hypothalamus - Eating disorders · Frontotemporal dementia $\cdot$ Volumetric MRI

\section{Introduction}

Behavioral variant frontotemporal dementia (bvFTD) is a neurodegenerative disorder characterized by atrophy in the frontal and temporal lobes and progressive behavioral and cognitive impairment [1]. Although the majority of cases are sporadic, about 10-20\% are due to an autosomal dominant mutation in one of three genes: microtubule-associated protein tau (MAPT), progranulin $(G R N)$, and chromosome 9 open reading frame 72 (C9orf72) [2]. One of the characteristic symptoms of bvFTD is the development of abnormal eating behaviors such as hyperphagia, changes in food preference, and craving for sweet foods, which are found in the majority of patients [3-5], and have 
been shown to help discriminate bvFTD from Alzheimer's disease $[6,7]$. However, the neuroanatomical correlates of abnormal eating behavior in bvFTD are unclear. Previous studies have suggested the importance of an orbitofrontalinsular-striatal brain network [8,9] but one study has also investigated the role of the hypothalamus, finding a correlation of abnormal eating behavior with lower volumes of the posterior hypothalamus [10].

The hypothalamus is the regulatory center for feeding and satiety [11]. It is composed of a number of different subnuclei and is highly interconnected with other parts of the central nervous system, particularly the brainstem, limbic system, and cortex [12, 13]. However, the hypothalamus is difficult to identify on magnetic resonance imaging and a detailed anatomical analysis of subdivisions of the hypothalamus has not yet been performed in bvFTD. In this study, we aimed to develop an optimized manual segmentation of the hypothalamus and its subunits using a novel protocol, and then use this to investigate patterns of atrophy in bvFTD and specifically whether differences could be seen in different genetic mutations.

\section{Methods}

\section{Participants}

Eighteen subjects fulfilling the criteria for the diagnosis of bvFTD [1] were recruited consecutively from a tertiary referral cognitive disorders clinic at the National Hospital for Neurology and Neurosurgery, London, UK. Nine subjects carried a mutation in the MAPT gene and 6 carried a pathogenic expansion in the $C 9$ orf 72 gene. The other three bvFTD subjects tested negative for mutations in $M A P T$, $C 9 o r f 72$, and GRN. Eighteen healthy controls were also recruited. Subjects' characteristics are summarized in Table 1. Written informed consent was obtained from all patients and controls, and the local ethics committee approved the study. Each patient underwent a standardized history and neurological examination (including assessment of function using the Frontotemporal dementia Rating Scale [14] ), neuropsychometry (including the MMSE), and assessment of behavioral symptoms using the Cambridge Behavioural Inventory Revised version (CBI-R) [15]. A subset of four questions on the CBI-R addresses the frequency of abnormal eating behavior scoring 0 for never occurring, 1 occurring a few times per month, 2 occurring a few times per week, 3 occurring daily, and 4 occurring constantly. The questions ask about whether sweet foods are preferred, whether the subject wants to eat the same foods repeatedly, whether their appetite is greater than before and whether there has been a decline in table manners. The total score for abnormal eating behavior was converted into percentage of impairment using methods described previously, where $1-50 \%$ is classified as mild or moderate, and $51-100 \%$ is classified as severe or very severe [16].

\section{Imaging parameters}

Volumetric T1- and T2-weighted MRI was performed in all 36 subjects. MRI scans were acquired on a $3 \mathrm{~T}$ scanner (Tim Trio, Siemens) with the following sequences: (i) high-resolution isotropic 3D T1-weighted MPRAGE (sagittal orientation; $\mathrm{TR}=2200 \mathrm{~ms}, \mathrm{TI}=900 \mathrm{~ms}, \mathrm{TE}=$ $2.9 \mathrm{~ms}$, flip angle $=10^{\circ}$, acquisition matrix $=256 \times 256$, and spatial resolution $=1.1 \mathrm{~mm}$ ) and (ii) high-resolution isotropic 3D T2-weighted fast spin echo/SPACE (sagittal orientation; TR $=3200 \mathrm{~ms}$, apparent $\mathrm{TE}=105 \mathrm{~ms}$, variable refocusing pulse flip angle to achieve T2-weighting, acquisition matrix $=256 \times 256$, and spatial resolution $=$ $1.1 \mathrm{~mm})$.

\section{Development of a hypothalamic segmentation protocol}

A review of hypothalamic anatomy and previously described hypothalamic segmentation protocols was made [10, 12, 13, 17-23]. The most detailed segmentation protocol described was by Schindler et al. [23] which itself had been designed following a survey of previously published protocols. However, in that study, they used 7T T1weighted MRIs which tend to be less widely available than 3T MRI. In order to optimize the protocol for 3T MRIs we made use of a volumetric T2-weighted MRI, acquired at the same time as the T1 image. By using the software package NiftyMIDAS (Centre for Medical Image Computing, UCL: http://cmic.cs.ucl.ac.uk/home/software/) which allows for the simultaneous viewing of different imaging modalities, we were able to perform a segmentation of the hypothalamus on registered volumetric T1- and T2-weighted images allowing better visualization of the boundaries of the hypothalamus (particularly laterally).

The segmentation protocol of Schindler et al. [23] was further optimized by reviewing descriptions of hypothalamic anatomy [17, 19-21] and criteria from other segmentation protocols $[10,12,13,18,22]$. Definitions of boundaries were made clearer, with greater detail provided in order to carefully include the hypothalamic nuclei in the segmentation (in particular the supraoptic, suprachiasmatic, retrochiasmatic nucleus, and the dorsal part of the arcuate (or infundibular) nucleus), and exclude the fornix. The protocol is defined in detail in the Supplementary Material. 
Table 1 Demographic, clinical, and behavioral variables for the bvFTD patients and controls

\begin{tabular}{lllll}
\hline & Controls & bvFTD & MAPT subgroup & C9orf72 subgroup \\
\hline Number of subjects & 18 & 18 & 9 & 6 \\
Gender, male & $9(50 \%)$ & $15(83.3 \%)$ & $7(77.8 \%)$ & $5(83.3 \%)$ \\
Age at scan (years) & $56.4(14.3)$ & $63.3(9.1)$ & $59.5(9.0)$ & $65.1(7.2)$ \\
Disease duration (years) & N/A & $9.1(5.5)$ & $8.0(5.6)$ & $10.8(6.4)$ \\
FRS (/100) & N/A & $33(24)$ & $38(26)$ & $28(25)$ \\
& & Range 3-73 & Range $7-73$ & Range 3-67 \\
Age at onset (years) & N/A & $54.3(8.5)$ & $51.4(6.3)$ & $54.3(9.8)$ \\
Education (years) & $14.2(3.0)$ & $14.3(4.3)$ & $14.2(4.8)$ & $13.3(3.9)$ \\
MMSE (/30) & $29.2(1.2)$ & $25.0(4.4)^{*}$ & $25.8(5.0)$ & $24.0(4.0) *$ \\
CBI-R Total (/180) & N/A & $76.5(31.8)$ & $76.4(36.9)$ & $78.7(33.4)$ \\
CBI-R eating disturbance score (/16) & N/A & $7.7(3.9)$ & $7.9(4.2)$ & $8.3(3.2)$ \\
CBI-R: "prefers sweet foods more than before" (/4) & N/A & $2.5(1.4)$ & $2.8(1.6)$ & $2.3(0.8)$ \\
CBI-R: "wants to eat the same foods repeatedly" (/4) & N/A & $2.1(1.5)$ & $2.6(1.5)$ & $1.3(1.4)$ \\
CBI-R: "her/his appetite is greater, s/he eats more than before" (/4) & N/A & $1.6(1.4)$ & $1.6(1.3)$ & $2.0(1.7)$ \\
CBI-R: "table manners are declining e.g., stuffing food into mouth" (/4) & N/A & $1.6(1.5)$ & $1.0(1.1)$ & $2.7(1.8)$ \\
\hline
\end{tabular}

Values denote mean (standard deviation) or $n(\%)$

$p$ values denote significance on Mann-Whitney $U$ or Chi square test

N/A not applicable, FRS frontotemporal dementia rating scale, $C B I-R$ Cambridge Behavioural Inventory Revised version

$* p<0.05$ disease group versus controls

\section{Methodology for segmentation}

Acquired T1-weighted images were initially transformed into standard space by a rigid registration to the Montreal Neurological Institute (MNI305) template [23-26]. Acquired T2 images were registered to the MNI305 template, using a transformation which combines the "T2 to T1native-space" and "T1 to MNI305-template" matrices (both derived after a six-parameter linear registration) using NiftyReg, revision \#418 (Centre for Medical Image Computing, UCL: http://cmic.cs.ucl.ac.uk/home/software/). Segmentations were performed manually on coronal slices using NiftyMIDAS. Segmentations were first performed on the T1 image, and then edited, switching to the corresponding T2weighted image which was superimposed on the $\mathrm{T} 1$.

\section{Reliability analysis}

The reliability of this optimized segmentation protocol was tested in a sample of ten cognitively normal controls, scanned using the same MRI protocol as the study participants. Hypothalamic structures were segmented twice. The intraclass correlation (ICC) was computed with a twoway random effects model, with Dice overlapping coefficients computed using the Convert3D tool (www.itksnap. org/pmwiki/pmwiki.php?n=Convert3D.Convert3D). The intrarater absolute intraclass correlation coefficient (ICC) was 0.931 (95\% confidence intervals: 0.723-0.983) and the Dice values were 0.88 (standard deviation 0.02) for both right and left hypothalamic segmentations, confirming excellent reliability of the protocol.

\section{Development of a hypothalamic subsegmentation protocol}

In order to investigate subregions of the hypothalamus in more detail, we adapted a methodology described by Makris et al. [21]. This uses visible anatomical landmarks on MRI scans to subsegment the hypothalamus into five subunits (Fig. 1): (i) the anterior superior hypothalamus (asHyp, which includes the paraventricular nucleus); (ii) the anterior inferior hypothalamus (a-iHyp, which includes the supraoptic nucleus); (iii) the superior tuberal hypothalamus (supTub, which includes the dorsomedial nucleus, the anterior part of the lateral hypothalamus, and the posterior part of the paraventricular nucleus); (iv) the inferior tuberal hypothalamus (infTub, which includes the arcuate (or infundibular) nucleus, the ventromedial nucleus and the posterior part of the supraoptic nucleus); and (v) the posterior hypothalamus (posHyp, which includes the posterior part of the lateral hypothalamus as well as the mammillary bodies). The detailed protocol for this subsegmentation is also included in the Supplementary Material.

\section{Methodology for subsegmentation}

Using hypothalamic segmentations defined above, delineation of the hypothalamic subunits was also performed 
Fig. 1 Segmentation of the hypothalamic subunits mapped on a 3T T1-weighted MR image of a control subject and their 3D reconstruction on a sagittal view. $a$-sHyp anterior superior hypothalamus, a-iHyp anterior inferior hypothalamus, supTub superior tuberal hypothalamus, infTub inferior tuberal hypothalamus, posHyp posterior hypothalamus

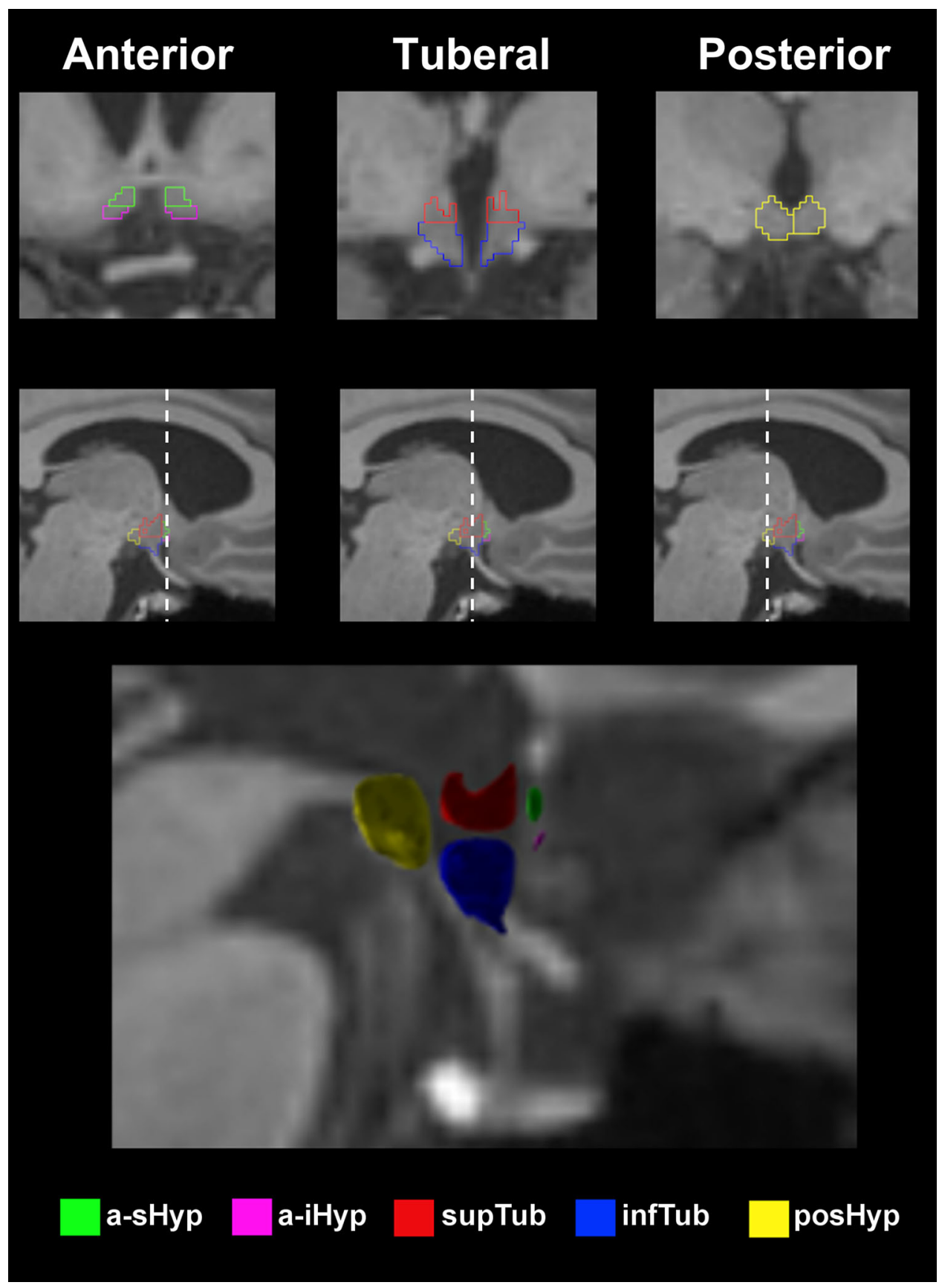

manually on coronal slices using NiftyMIDAS. This was performed initially on the T1 image, switching to the corresponding superimposed $\mathrm{T} 2$ image for editing.

\section{Calculation of hypothalamic volumes}

Volumes of the whole hypothalamus and its subunits were automatically computed from the segmentations performed in NiftyMIDAS and corrected for total intracranial volume (TIV), which was calculated using the Statistical
Parametric Mapping (SPM) 12b software, version 5953 (www.fil.ion.ucl.ac.uk/spm), running under Matlab R2012a (Math Works, Natick, MA, USA). The TIV corrected volume of a specific structure (i.e., the hypothalamus or a subunit) for each subject "i" was computed as follows: Structure volume $_{\text {corrected(i) }}=$ Structure volume $_{\text {raw(i) }} *_{-}$

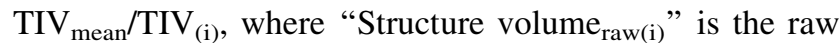
value of the structure of the subject " $i$," "TIV $V_{\text {mean }}$ is the average TIV of the study group, and "TIV $(\mathrm{i})$ " is the TIV of the subject "i." 


\section{Statistical analyses}

Statistical analyses were performed in SPSS software (SPSS Inc., Chicago, IL, USA) version 12.0 and in R language v.3.0.2. Differences in demographic, cognitive, and clinical features as well as brain volumes were tested with the Mann-Whitney $U$ test for continuous variables (due to the small sample size and to the non-normal distribution for all the variables) and Chi square test for dichotomous variables. For the hypothalamic subunit volumetry (five subunits on either side), a correction for multiple comparisons was made so that only a threshold of $p<0.005$ was considered significant.

\section{Results}

Demographic characteristics are reported in Table 1: no significant differences were found in gender, age, or education between controls and bvFTD, but the patient group scored significantly lower on the MMSE. All patients scored abnormally on the eating disturbance subscale of the CBI-R: 12 patients scored in the mild to moderate range $(1-50 \%)$ and 6 patients scored in the severe to very severe range $(51-100 \%)$.

No significant differences were seen in demographics, disease duration, MMSE, FRS, or total CBI-R score between the MAPT and C9orf72 subgroups. Looking at the individual eating disturbance subscores of the CBI-R, there was a trend for higher scores in the MAPT group compared with $C 9$ orf72 in wanting to eat the same foods repeatedly [mean (SD) score 2.6 (1.3) versus 1.3 (1.4)], with the opposite trend (higher score in C9orf72) in decline in table manners [mean (SD) score 2.7 (1.8) versus 1.0 (1.1)].

The bvFTD group showed a $17 \%$ lower total hypothalamic volume compared with controls (mean (SD) 783 (113) versus 944 (73) $\mathrm{mm}^{3}, p<0.0005$, MannWhitney $\mathrm{U}$ test) with a similar reduction in both the right $(17 \%)$ and left $(18 \%)$ hypothalamus compared with controls. The MAPT mutation group showed a non-significant lower right and left hypothalamic volumes on both sides compared with C9orf72 (10-13\% difference) (Table 2).

The subsegmentation analysis revealed significant differences in the total (left and right combined) volumes of the superior regions (both anterior and tuberal) as well as the posterior region (superior tuberal $22 \%$, posterior $24 \%$, and anterior superior region $41 \%$ smaller than controls), with a similar pattern when looking at the individual right and left volumes. No significant differences survived correction for multiple comparisons in the inferior regions (anterior and tuberal) between the bvFTD group and controls (Table 2).
Looking at the individual MAPT and $C 9$ orf 72 groups, only the MAPT mutation carriers showed significant differences from controls when corrected for multiple comparisons with superior (anterior and tuberal) and posterior regions being smaller. The $C 9$ orf 72 group showed a trend to smaller anterior superior $(p=0.009)$ and left superior tuberal regions $(p=0.047)$. Direct comparisons of the genetic groups revealed a trend for a smaller right superior tuberal region $(16 \%, p=0.036)$ and posterior region $(24 \%, p=0.066)$ in the MAPT group compared with C9orf72.

In the total bvFTD group, patients who scored in the severe to very severe range of the CBI-R eating disturbance subscale had a trend to a lower total hypothalamic volume [740 (89) $\left.\mathrm{mm}^{3}\right]$ than those in the mild to moderate range [805 $\left.(122) \mathrm{mm}^{3}\right]$. This trend for a lower volume in those scoring in the severe to very severe range was also seen in the superior tuberal region [205 (36) versus $236(37) \mathrm{mm}^{3}$ ]. Similarly, in both genetic groups, there was a non-significantly lower total hypothalamic volume in those scoring within the severe to very severe range: in the MAPT group 721 (120) versus 773 (147) $\mathrm{mm}^{3}$, in the C9orf72 group 794 (35) versus $884(60) \mathrm{mm}^{3}$.

\section{Discussion}

Using a novel segmentation protocol for the hypothalamus and its subunits based upon registered volumetric $\mathrm{T} 1$ and T2 MR images, we have shown that the hypothalamus is substantially smaller in patients with bvFTD compared with controls, particularly in the superior and posterior regions. There was also a trend for a smaller hypothalamus, particularly in the superior tuberal region, in those who had severe eating disturbance. Furthermore, there is significant atrophy in the MAPT mutation group (in superior and posterior areas), but no significant differences from controls in the C9orf72 mutation group.

Our findings are different from the only previous study of hypothalamic volume in FTD [10], which found significant atrophy in the posterior hypothalamus. Our study differed from this in a number of aspects, both technical and clinical. Technically, we used different criteria for the segmentation of the hypothalamus, in particular, paying attention to the exclusion of the fornix, and inclusion of the supraoptic, suprachiasmatic, retrochiasmatic, and arcuate (or infundibular) nuclei. Furthermore, we were able to delineate five specific subunits of the hypothalamus, whereas Piguet et al. [10] used an arbitrary definition of anterior and posterior regions of the hypothalamus, splitting it through the middle coronal plane of their segmentation. Their 'posterior' hypothalamus may therefore include part of the tuberal region as we have defined it in 
Table 2 Volumetry of hypothalamus and its subunits in 18 bvFTD (including nine MAPT and six C9orf 72 mutation carriers) and 18 control subjects

\begin{tabular}{|c|c|c|c|c|c|c|}
\hline & Controls & bvFTD & $\begin{array}{l}\% \text { difference } \\
\text { (negative means } \\
\text { smaller in bvFTD } \\
\text { than controls) }\end{array}$ & $M A P T$ subgroup & C9orf72 subgroup & $\begin{array}{l}\% \text { difference } \\
\text { (negative means } \\
\text { smaller in } M A P T \\
\text { than } C 9 \text { orf } 72 \text { ) }\end{array}$ \\
\hline Hypothalamus-total & $944(73)$ & $783(113)^{* *}$ & -17 & $756(133)^{* *}$ & $854(68)^{*}$ & -11 \\
\hline Hypothalamus_right & $477(38)$ & $398(62) * *$ & -17 & $380(73)^{* *}$ & $436(41)^{*}$ & -13 \\
\hline Hypothalamus-left & $467(39)$ & $385(53) * *$ & -18 & $375(63)^{* *}$ & $418(31)^{*}$ & -10 \\
\hline Anterior superior-total & $46(18)$ & $27(13)^{* *}$ & -41 & $25(13) * *$ & $25(11)^{*}$ & 0 \\
\hline Anterior superior-right & $22(10)$ & $13(7)^{* *}$ & -43 & $12(6)^{*}$ & $12(7)^{*}$ & 0 \\
\hline Anterior superior-left & $23(9)$ & $14(6)^{* *}$ & -38 & $13(7)^{*}$ & $14(5)^{*}$ & -7 \\
\hline Anterior inferior-total & $30(18)$ & $18(8)$ & -40 & $20(8)$ & $17(8)$ & +18 \\
\hline Anterior inferior-right & $15(10)$ & $9(4) *$ & -40 & $8(4)$ & $8(4)$ & 0 \\
\hline Anterior inferior-left & $15(8)$ & $10(5)$ & -34 & $11(5)$ & $8(5)$ & +38 \\
\hline Superior tuberal-total & $289(54)$ & $225(38) * *$ & -22 & $213(41)^{* *}$ & $251(30)$ & -15 \\
\hline Superior tuberal—right & $145(30)$ & $114(20)^{* *}$ & -21 & $108(18)^{* *, \wedge}$ & $129(19)$ & -16 \\
\hline Superior tuberal-left & $144(24)$ & $111(20)^{* *}$ & -23 & $105(25)^{* *}$ & $122(12)^{*}$ & -14 \\
\hline Inferior tuberal—total & $317(38)$ & $314(37)$ & -1 & $317(48)$ & $322(22)$ & -2 \\
\hline Inferior tuberal—right & $162(20)$ & $158(20)$ & -2 & $158(26)$ & $163(13)$ & -3 \\
\hline Inferior tuberal—left & $155(22)$ & $156(19)$ & +1 & $159(23)$ & $159(11)$ & 0 \\
\hline Posterior-total & $263(49)$ & $199(59)^{* *}$ & -24 & $181(60)^{* *}$ & 239 (49) & -24 \\
\hline Posterior-right & $133(27)$ & $104(34)^{*}$ & -22 & $94(35) * *$ & 124 (29) & -24 \\
\hline Posterior-left & $130(24)$ & $95(27)^{* *}$ & -26 & $87(26) * *$ & $115(26)$ & -24 \\
\hline
\end{tabular}

Volumes are corrected for TIV. Values denote mean (standard deviation) volumes in $\mathrm{mm}^{3} . p$ values denote significance on Mann-Whitney $U$ test $* p<0.05$, ** $p<0.005$ disease group versus controls; ${ }^{\wedge} p<0.05$ MAPT versus C9orf72 subgroups. Significance threshold was set at $p<0.005$ to correct for multiple comparisons

this study. Clinically, it is unclear whether the cohorts overlap as their 18 patients are not genetically defined unlike the group studied here which contains a significant number of genetic bvFTD cases. The Piguet cohort has an earlier mean disease duration (3.3 years versus 9.1 years here), although disease severity is similar (MMSE 23.9 versus 25.0 here; CBI-R eating disturbance score 6.9 versus 7.7 here). In a separate pathological analysis, they investigated six tau-positive and six TDP-43 positive FTD cases, also finding problems more posteriorly, attributing this to atrophy in the TDP-43 group. However, their tau group contained only cases with a specific type of tau pathology, Pick's disease (a 3-repeat tauopathy), and their TDP-43 group similarly contained only cases with one subtype (TDP-43 type B). Recent studies have made it clear that there are large differences both clinically and pathologically between the different tau and TDP-43 pathological subtypes (which number at least four in each group), and so one cannot extrapolate to a significant difference between 'all TDP-43' and 'all tau' pathology by investigating only one subtype. Our study suggests that a particular tau group (MAPT mutations) appear to have significant hypothalamic volume loss compared with a TDP-43 group (C9orf72 expansions), the opposite finding to Piguet et al. [10].
We found that patients with more severe eating disturbance had a trend to lower hypothalamic volumes, particularly within the superior tuberal region. However, the differences did not reach statistical significance and so caution should be attributed to these findings until further studies are performed. The CBI-R provides only limited information on the many different types of abnormal eating behaviors, as only four questions are asked. Eating behaviors in bvFTD are complex and varied, and include different aspects, such as carbohydrate craving, overeating, obsessions for specific foods and oral exploration of inedible objects, which may not always coexist in an individual patient $[7,27,28]$. There was a trend for a difference in the type of eating behaviors exhibited between the two genetic groups with the MAPT group scoring higher on wanting to eat the same foods repeatedly and the C9orf72 group showing a greater decline in table manners. Further studies are required using more detailed feeding questionnaires to explore these issues further [7].

This study found significant atrophy in the superior and posterior subunits of the hypothalamus which contain the paraventricular nucleus (anterior superior region), dorsomedial nucleus (superior tuberal region), and lateral hypothalamic areas (superior tuberal and posterior 
regions). These subnuclei are all involved in important aspects of appetite regulation and contain neuropeptideexpressing neurons and neuropeptide receptors [20, 2932]. Interestingly, the inferior tuberal area was not atrophic, an area which contains the arcuate (or infundibular) and ventromedial nuclei. The arcuate nucleus is the primary target of metabolic and hormonal signals from the periphery, with important connections to other nuclei in the hypothalamus particularly the paraventricular nucleus [30]. This suggests that appetite abnormalities in bvFTD could be due to changes in neuropeptides (or neuropeptide receptors) within the superior and posterior areas of the hypothalamus and/or from disruption of pathways from the arcuate nucleus to other areas of the hypothalamus. As there is differential neuropeptide expression within these nuclei, different neuropeptide levels should be impaired compared with others in bvFTD: such a hypothesis has yet to be explored.

There was a trend for greater posterior involvement in the MAPT group compared with the C9orf72 group, with both left and right posterior regions $24 \%$ smaller in the MAPT group. As well as the posterior part of the lateral hypothalamus, the posterior region contains the mammillary bodies, which are connected to the amygdala and hippocampus, areas known to be major areas of atrophy in patients with MAPT mutations [33]. Given this finding, future studies should further investigate the relationship of memory impairment in these patients to hypothalamic atrophy.

In summary, bvFTD patients had smaller hypothalamic volumes compared with controls, with atrophy localized to subnuclei regulating food intake, reward and perception of satiety, and related to the severity of the eating disturbance. Moreover, different genetic mutations seem to have a differential impact on the hypothalamus, although further studies in larger genetic and pathological series are required to confirm this. The structural and functional connections of the hypothalamus should be further explored in bvFTD, particularly how they relate to the orbitofrontal-insular-striatal reward network previously identified. Lastly, the results of this study suggest testable hypotheses of the role of different neuropeptides in impaired appetite regulation in bvFTD.

Acknowledgments This work was funded by the Medical Research Council, UK and Alzheimer's Research UK. The authors acknowledge the support of the NIHR Queen Square Dementia Biomedical Research Unit, Leonard Wolfson Experimental Neurology Centre, and the University College London Hospitals NHS Trust Biomedical Research Centre. The Dementia Research Centre is an Alzheimer's Research UK coordinating centre and has also received equipment funded by Alzheimer's Research UK and Brain Research Trust. JDR is a NIHR Rare Disease TRC Postdoctoral Fellow. JDW is supported by a Wellcome Trust Senior Clinical Fellowship (091673/Z/10/Z). MNR is a NIHR Senior Investigator. JB and EM are funded by
Alzheimer's Research UK. SO is funded by the Engineering and Physical Sciences Research Council (EP/H046410/1, EP/J020990/1, EP/K005278), the Medical Research Council (MR/J01107X/1), the EU-FP7 project VPH-DARE@IT (FP7- ICT-2011-9-601055), and the National Institute for Health Research University College London Hospitals Biomedical Research Centre (NIHR BRC UCLH/UCL High Impact Initiative BW.mn.BRC10269. MM is supported by the UCL Leonard Wolfson Experimental Neurology Centre (PR/ylr/ 18575).

\section{Compliance with ethical standards}

Conflict of interest Dr. Rossor is a National Institute for Health Research Senior Investigator; he also reports fees (paid to University College London) for serving on a Data Monitoring Committee for Servier outside the submitted work. Dr. Frisoni has served in advisory boards for Lilly, BMS, Bayer, Lundbeck, Elan, Astra Zeneca, Pfizer, Taurx, Wyeth, GE, Baxter. Dr. Frisoni is member of the editorial boards of Lancet Neurology, Aging Clinical and Experimental Research, Alzheimer's Diseases and Associated Disorders, and Neurodegenerative Diseases, and Imaging Section Editor of Neurobiology of Aging. Dr. Frisoni has received grants from Wyeth Int.l, Lilly Int.1, Lundbeck Italia, GE Int.1, Avid/Lilly, Roche, Piramal, and the Alzheimer's Association. Research of industrial interest has touched memantine, PET amyloid ligands, diagnostic and tracking Alzheimer's biomarkers, and memantine. In the last 2 years, Dr. Frisoni has received fees for lectures as an invited speaker from Lundbeck, Piramal, and GE. All other authors report no disclosures.

Ethical standard This study was approved by the London Queen Square Research Ethics Committee.

Open Access This article is distributed under the terms of the Creative Commons Attribution 4.0 International License (http://crea tivecommons.org/licenses/by/4.0/), which permits unrestricted use, distribution, and reproduction in any medium, provided you give appropriate credit to the original author(s) and the source, provide a link to the Creative Commons license, and indicate if changes were made.

\section{References}

1. Rascovsky K, Hodges JR, Knopman D, Mendez MF, Kramer JH, Neuhaus J et al (2011) Sensitivity of revised diagnostic criteria for the behavioural variant of frontotemporal dementia. Brain 134:2456-2477

2. Warren JD, Rohrer JD, Rossor MN (2013) Clinical review. frontotemporal dementia. BMJ 347:f4827

3. Diehl-Schmid J, Pohl C, Perneczky R, Forstl H, Kurz A (2006) Behavioral disturbances in the course of frontotemporal dementia. Dement Geriatr Cogn Disord 22:352-357

4. Piguet O, Hornberger M, Shelley BP, Kipps CM, Hodges JR (2009) Sensitivity of current criteria for the diagnosis of behavioral variant frontotemporal dementia. Neurology 72:732-737

5. Piguet O (2011) Eating disturbance in behavioural-variant frontotemporal dementia. J Mol Neurosci 45:589-593

6. Bozeat S, Gregory CA, Ralph MA, Hodges JR (2000) Which neuropsychiatric and behavioural features distinguish frontal and temporal variants of frontotemporal dementia from Alzheimer's disease? J Neurol Neurosurg Psychiatry 69:178-186

7. Ikeda M, Brown J, Holland AJ, Fukuhara R, Hodges JR (2002) Changes in appetite, food preference, and eating habits in 
frontotemporal dementia and Alzheimer's disease. J Neuro Neurosurg Psychiatry 73:371-376

8. Whitwell JL, Sampson EL, Loy CT, Warren JE, Rossor MN, Fox NC et al (2007) VBM signatures of abnormal eating behaviours in frontotemporal lobar degeneration. NeuroImage 35:207-213

9. Woolley JD, Gorno-Tempini ML, Seeley WW, Rankin K, Lee SS, Matthews BR et al (2007) Binge eating is associated with right orbitofrontal-insular-striatal atrophy in frontotemporal dementia. Neurology 69:1424-1433

10. Piguet O, Petersen A, Yin Ka Lam B, Gabery S, Murphy K, Hodges JR et al (2011) Eating and hypothalamus changes in behavioral-variant frontotemporal dementia. Ann Neurol 69:312-319

11. Morton GJ, Cummings DE, Baskin DG, Barsh GS, Schwartz MW (2006) Central nervous system control of food intake and body weight. Nature 443:289-295

12. Baroncini M, Jissendi P, Balland E, Besson P, Pruvo JP, Francke JP et al (2012) MRI atlas of the human hypothalamus. Neuroimage 59:168-180

13. Goldstein JM, Seidman LJ, Makris N, Ahern T, O'Brien LM, Caviness VS Jr et al (2007) Hypothalamic abnormalities in schizophrenia: sex effects and genetic vulnerability. Biol Psychiatry 61:935-945

14. Mioshi E, Hsieh S, Savage S, Hornberger M, Hodges JR (2010) Clinical staging and disease progression in frontotemporal dementia. Neurology 74:1591-1597

15. Wear HJ, Wedderburn CJ, Mioshi E, Williams-Gray $\mathrm{CH}$, Mason SL, Barker RA et al (2008) The Cambridge Behavioural Inventory Revised. Dementia Neuropsychologia 2:102-107

16. Lillo P, Mioshi E, Zoing MC, Kiernan MC, Hodges JR (2011) How common are behavioural changes in amyotrophic lateral sclerosis? Amyotroph Lateral Scler. 12(1):45-51

17. Daniel PM (1976) Anatomy of the hypothalamus and pituitary gland. J Clin Pathol Suppl (Assoc Clin Pathol) 7:1-7

18. Gabery S, Georgiou-Karistianis N, Lundh SH, Cheong RY, Churchyard A, Chua P et al (2015) Volumetric Analysis of the Hypothalamus in Huntington Disease Using 3T MRI: the IMAGE-HD Study. PLoS One 10(2):e0117593

19. Jacobson S, Marcus EM. Neuroanatomy for the Neuroscientist. Springer, 2011

20. Lang J (1985) Surgical anatomy of the hypothalamus. Acta Neurochir (Wien) 75:5-22
21. Mai JK, Assheuer J, Paxinos G (1997) Atlas of the human brain. Academic Press, San Diego CA

22. Makris N, Swaab DF, van der Kouwe A, Abbs B, Boriel D, Handa RJ et al (2013) Volumetric parcellation methodology of the human hypothalamus in neuroimaging: normative data and sex differences. NeuroImage 69:1-10

23. Schindler S, Schonknecht P, Schmidt L, Anwander A, Strauss M, Trampel R et al (2013) Development and evaluation of an algorithm for the computer-assisted segmentation of the human hypothalamus on 7-tesla magnetic resonance images. PLoS One 8:e66394

24. Evans AC, Collins DL, Milner B (1992) An MRI-based stereotaxic atlas from 250 young normal subjects. Proc 22nd Annual Symposium, Society for. Neuroscience 18:408

25. Evans AC, Marrett S, Neelin P, Collins L, Worsley K, Dai W et al (1992) Anatomical mapping of functional activation in stereotactic coordinate space. NeuroImage. 1:43-63

26. Evans AC, Collins DL, Mills SR, Brown ED, Kelly RL, Peters TM (1993) 3D statistical neuroanatomical models from 305 MRI volumes. Proc IEEE Nuclear Science Symposium and Medical Imaging Conference pp 1813-1817

27. Miller BL, Darby AL, Swartz JR, Yener GG, Mena I (1995) Dietary changes, compulsions and sexual behavior in frontotemporal degeneration. Dementia 6:195-199

28. Rosen HJ, Allison SC, Schauer GF, Gorno-Tempini ML, Weiner MW, Miller BL (2005) Neuroanatomical correlates of behavioural disorders in dementia. Brain 128:2612-2625

29. Coll AP, Farooqi IS, O'Rahilly S (2007) The hormonal control of food intake. Cell 129:251-262

30. Parker JA, Bloom SR (2012) Hypothalamic neuropeptides and the regulation of appetite. Neuropharmacology 63:18-30

31. Saper CB, Chou TC, Elmquist JK (2002) The need to feed: homeostatic and hedonic control of eating. Neuron 36:199-211

32. Woods SC, Schwartz MW, Baskin DG, Seeley RJ (2000) Food intake and the regulation of body weight. Annu Rev Psychol 51:255-277

33. Rohrer JD, Ridgway GR, Modat M, Ourselin S, Mead S, Fox NC et al (2010) Distinct profiles of brain atrophy in frontotemporal lobar degeneration caused by progranulin and tau mutations. NeuroImage. 53(3):1070-1076 